\title{
2-Octyl Cyanoacrylate Glue Application on Split-Thickness Skin Graft for Lower Extremity Reconstruction
}

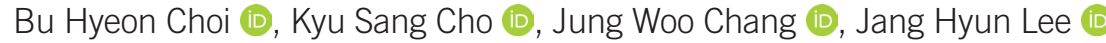 \\ Department of Plastic and Reconstructive Surgery, Hanyang University Guri Hospital, Hanyang University College of Medicine, Guri, Korea
}

\begin{abstract}
A split-thickness skin graft (STSG) is a very effective method for reconstructing skin defects in the lower extremities; however, suturing all graft margins during surgery is time-consuming. As an alternative, 2-octyl cyanoacrylate glue attaches the epidermal layer of an STSG to normal skin and functions like a suture. In this study, we assessed 2-octyl cyanoacrylate glue as a time-saving step in STSG procedures. We reviewed the charts of 87 patients who underwent STSG between May 2018 and August 2020. For the 10 patients who consented to the STSG procedure with 2-octyl cyanoacrylate glue, we evaluated the treatment site for complications and engraftment every 2 days for 14 days. The STSGs were successfully engrafted in all cases. Seroma, hematoma, wound dehiscence, or infection were not documented. No additional revision surgery was required. The use of 2-octyl cyanoacrylate glue in STSG margin fixation was safe, effective, convenient, and time-saving. This study found 2-octyl cyanoacrylate glue a good option for lower extremity skin defect reconstruction.
\end{abstract}

Keywords: 2-Octyl cyanoacrylate; Reconstructive surgical procedures; Skin transplantation

\section{Introduction}

Clinicians have long investigated methods to repair skin defects. While skin injuries, such as lacerations, are primarily closed with sutures, large skin defects may require repair with flaps or skin grafts. Skin grafts are occasionally preferred to flaps because they require shorter operations; however, suturing the entire graft margin is still timeconsuming [1]. Several techniques have been proposed for reducing operating times, including applying negative pressure wound therapy and using skin staplers [1-3]. Meanwhile, the practice of applying 2-octyl cyanoacrylate glue (Dermabond; Ethicon Inc., Somerville, NJ, USA) onto skin grafts has not been extensively studied. Dermabond binds the epidermal layer of the graft to normal skin and functions similarly to sutures.

The authors examined the advantages and disadvantages of using Dermabond in patients who underwent split-thickness skin graft (STSG) surgery for skin defect repair.

\section{Ideas}

\section{Selection criteria}

A retrospective review of 87 patients who underwent STSG between May 2018 and August 2020 was performed. Patients with incomplete medical records, less than 6
Ideas and Innovations

Received: February 20, 2021

Revised: August 14, 2021

Accepted: August 15, 2021

Corresponding author: Jang Hyun Lee, M.D., Ph.D.

Department of Plastic and Reconstructive Surgery, Hanyang University Guri Hospital, Hanyang University College of Medicine, 153 Gyeongchunro, Guri 11923, Korea

Tel: +82-31-560-2330

Fax: +82-31-560-2338

E-mail: pslee1999@hanmail.net

This is an Open Access article distributed under the terms of the Creative Commons Attribution Non-Commercial License (https://creativecommons.org/licenses/by-nc/4.0/) which permits unrestricted non-commercial use, distribution, and reproduction in any medium, provided the original work is properly cited.

(c) 2021 Korean Wound Management Society 
months of follow-up, and in whom skin staplers or negative pressure wound therapy were applied $(n=67)$ were excluded from this study. The patients were provided written information regarding the details of the procedure. Patients were subsequently assigned to the Dermabond group if they provided consent for Dermabond application in lower extremity skin defect reconstruction. The study was performed in accordance with the principles of the Declaration of Helsinki. Ten patients with skin defects in the lower extremities underwent STSG with Dermabond application at our hospital.

\section{Application of Dermabond}

Before the STSG operation, infection of the wound was ruled out based on the wound's clinical presentation or a skin culture test. The wound was also assessed to confirm that there was no tissue exposure such as bone or tendon which would interfere with engraftment of the STSG.

During the operation, the wound bed was prepared aseptically. Skin grafts were usually harvested from the thigh at a thickness of 12/1,000 inches using a Zimmer dermatome. Slit incisions were made to allow for potential hematoma drainage. Grafts were trimmed to cover the wound size with an excess margin of $2 \mathrm{~mm}$. Dermabond was applied to the excess 2-mm margin of the normal skin around the wound as one would apply glue. Next, the skin graft was placed over and fixed to the wound and the excess margin. In some cases, basting sutures were placed for graft fixation. Postoperatively, the wound was dressed with Bactigras (Smith \& Nephew, Wat- ford, UK) and ointment. Bactigras and ointment were carefully applied only on the wound, avoiding the margin as their contact with the margin could reduce the holding effect of Dermabond. Mild compression dressing was performed using gauze and elastic bandage.

\section{Postoperative management}

Skin grafts were monitored every 2 days. The grafts were monitored closely to identify any signs of infection, hematoma, or other factors that would interrupt graft taking. The un-taken excess graft margins were trimmed where Dermabond was applied on the 7th day. On the 14th postoperative day, the graft success and complications of the STSG was evaluated through inspection by all authors. Complications included seroma, hematoma, infection, and whether additional revision surgery was required.

\section{Outcomes of Dermabond application}

All skin grafts were well engrafted on the wound bed. There were no cases of seroma, hematoma, wound dehiscence, or infection. No additional revision surgery was required. In this study, we retrospectively review 10 cases to analyze our Dermabond-applying method, and of those 10 , present two representative cases in detail.

\section{Case 1}

An 18-year-old healthy male sustained a laceration to the dorsum of his left foot from a motorcycle accident. He underwent
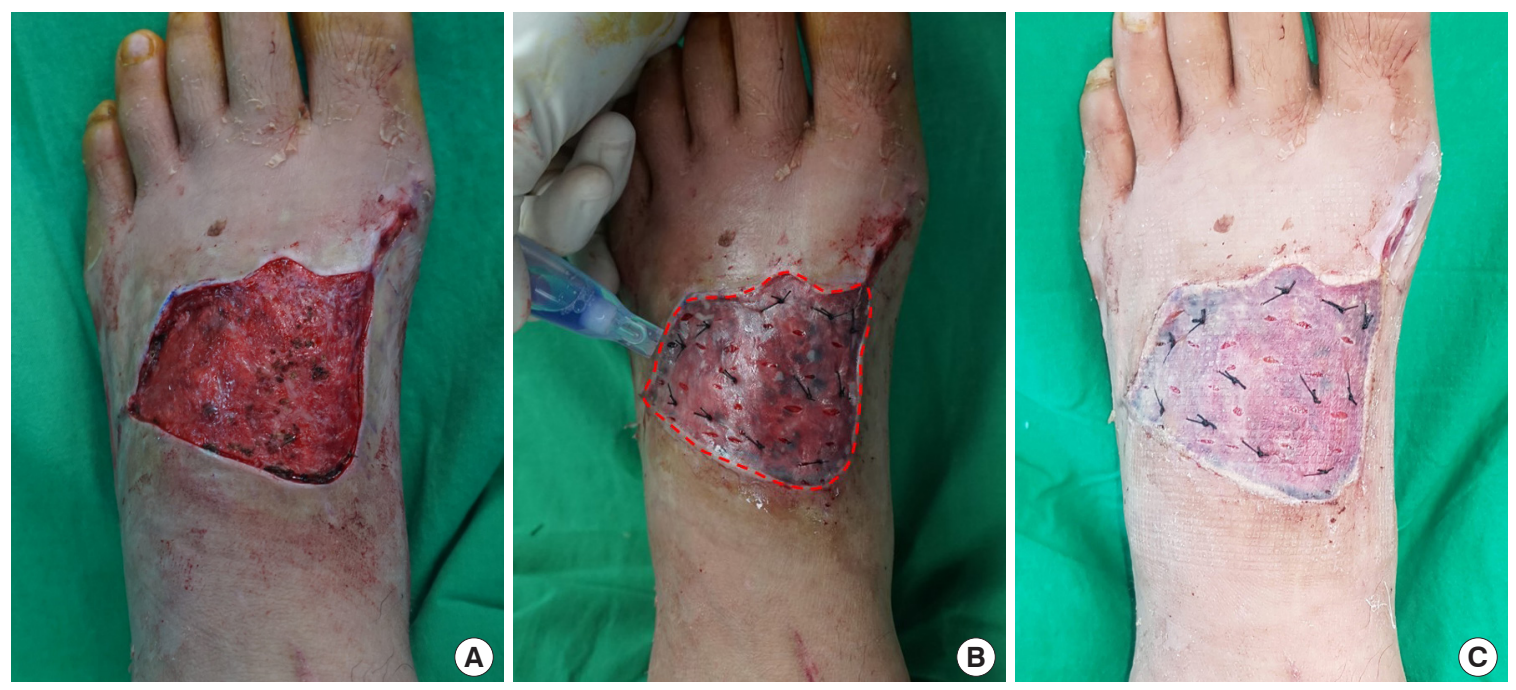

Fig. 1. Application of Dermabond to the dorsum of the left foot. (A) Preoperative photograph of a trauma-induced skin defect on the dorsum of the left foot of an 18-year-old male. (B) Dermabond (2-octyl cyanoacrylate glue) is applied along the red dashed line between the excess graft margin and normal skin around the wound. (C) Condition of the wound on the 7th postoperative day. 
primary closure at our institution, but the wound gradually darkened and demonstrated signs of necrosis. The wound was treated with aseptic dressings for 1 month but did not show improvement, and the patient was admitted for surgical treatment. A bacterial culture test on the wound bed was performed, and an empirical intravenous antibiotic was administered. The culture test demonstrated no growth. STSG was performed under general anesthesia to repair the skin defect. The necrotic tissue was debrided, and the final skin defect measured $8 \times 6 \mathrm{~cm}$ (Fig. 1A). Dermabond was applied along the red dashed line between the excess graft margin and the normal skin around the wound (Fig. 1B). Mild compression dressing was applied with gauze and elastic bandages. The operating time was 40 minutes. On the 7 th postoperative day, the excess graft margins that did not take were trimmed. The STSG was well-taken and no signs of hematoma, seroma, or infection were observed (Fig. 1C).

\section{Case 2}

A 62-year-old female with underlying diabetes mellitus and hypertension visited our institution for deep second-degree burns on the dorsum of the right foot caused by hot water. After debridement of the necrotic tissue, the skin defects measured $3 \times 5 \mathrm{~cm}$ and $1 \times 1 \mathrm{~cm}$. There was no bone or tendon exposure. The margins of the STSGs were fixed with Dermabond under local anesthesia (Fig. 2A). The operating time was 25 minutes. On the 7th postoperative day, both STSGs ap- peared stable and did not show any complications (Fig. 2B). On the 14th postoperative day, both STSGs were well-taken on the wound bed (Fig. 2C).

\section{Discussion}

Dermabond is currently used in many operations. Sutureless circumcision operations are a typical application, and other examples include breast surgery, thyroid cancer, cleft lip, and knee arthroplasty operations [4-7].

Several studies have demonstrated that cosmesis and patient satisfaction with Dermabond was not significantly different from that of sutures while Dermabond shortened operating times [7-9]. Utilizing Dermabond in pediatric procedures may be advantageous because it is difficult to maintain aseptic dressings in these patients. An application of Dermabond over the site also prevents bacterial infection $[6,10]$.

The role of Dermabond in skin graft surgery has not been sufficiently studied. As a preliminary test, Sivrioglu et al. [11] utilized Dermabond to fix STSGs in urogenital tissue defects. To our knowledge, this study was the first to perform reconstruction using Dermabond with STSG in the lower extremities.

As STSGs are very thin, they are prone to damage when the surgeon applies the very sticky Dermabond and manipulates the graft. Therefore, as presented in this study, damage and loss of the skin graft can be prevented by applying Dermabond
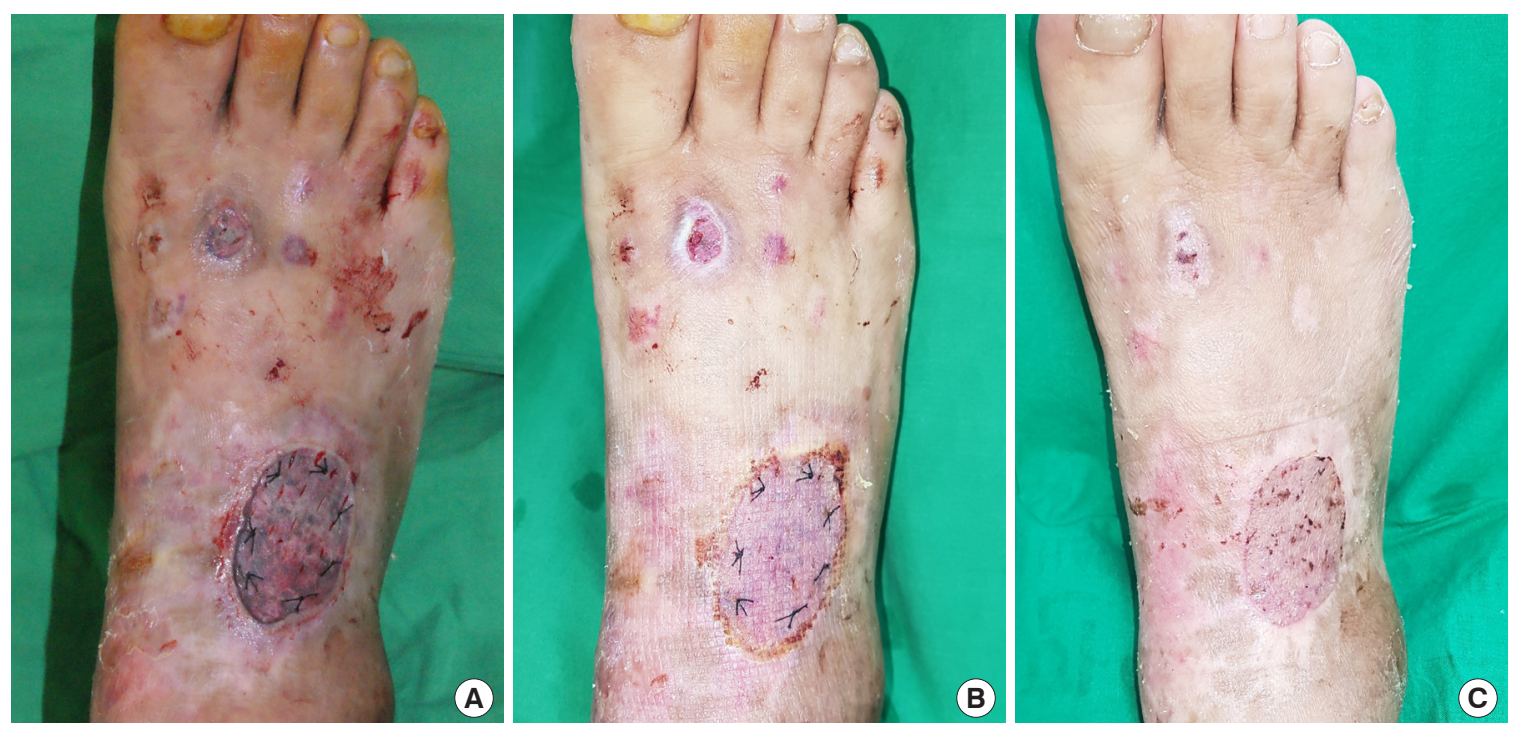

Fig. 2. Immediate postoperative and outpatient follow-up photographs. (A) Immediate postoperative condition of a second-degree burn on the dorsum of the right foot of a 62-year-old female. (B) Condition of the wound on the 7th postoperative day. (C) Condition of the wound 2 weeks after the skin graft. 
only between an excess graft margin of $2 \mathrm{~mm}$ and the nomal skin around the wound.

While skin grafts require relatively shorter operations, skin graft procedures are still time-consuming. When sutures are utilized for graft fixation, operating times naturally increase in proportion with the size of the graft. In contrast, Dermabond application requires 5-30 seconds regardless of the size of the graft; its potential for time-saving is clear. Moreover, many skin graft operations are performed under general anesthesia. Applying Dermabond may help reduce anesthesia-related complications from extended operations [12].

Skin staplers are also utilized in STSG surgery and require relatively shorter operations; however, skin staples may become buried, which provides a path for bacterial infection. Skin staples may also pull on the graft margins, resulting in graft disfigurement. Staple removal may also be painful. In contrast, Dermabond application does not require suture removal [3]. The authors found no notable difference in graft success and complications between the suture- and Dermabond-based procedures. The esthetic results at the outpatient follow-up visits were also similar. Our data demonstrated that Dermabond application on skin grafts for lower extremity reconstruction was safe, effective, and time-saving.

Nevertheless, this study has the following limitations. The sample size was small and did not account for other factors that may affect wound healing, such as glucocorticoid steroid medication, immunocompromised conditions, and vascular patency [13]. Meanwhile, though our study was also limited to the lower extremities, our data can likely be applied to the reconstruction of skin defects in the upper extremities. In addition, although our study analyzed non-meshed STSG, our data may be applied to meshed STSG if the skin defect exceeds the available healthy donor sites.

In conclusion, this study showed that Dermabond shortened operating times and had no difference in terms of complications and graft success when compared to the conventional method. Dermabond application can therefore be considered in lower extremity skin defect reconstruction.

\section{Conflict of interest}

Jung Woo Chang and Jang Hyun Lee are editorial board members of the journal but were not involved in the peer reviewer selection, evaluation, or decision process of this article. No other potential conflicts of interest relevant to this article were reported.

\section{ORCID iDs}

Bu Hyeon Choi

Kyu Sang Cho

Jung Woo Chang

Jang Hyun Lee

https://orcid.org/0000-0003-4171-1303

https://orcid.org/0000-0002-3235-1548

https://orcid.org/0000-0002-7937-9679

https://orcid.org/0000-0002-0552-4554

\section{References}

1. Inatomi Y, Kadota H, Kamizono K, et al. Securing splitthickness skin grafts using negative-pressure wound therapy without suture fixation. J Wound Care 2019;28(Suppl 8): S16-21.

2. Shen X, Zhan T, Wei D, et al. Comparison of efficacy and complications between negative pressure wound therapy and conventional mechanical fixation in skin grafts: a retrospective analysis. Wounds 2019;31:213-8.

3. Tajirian AL, Goldberg DJ. A review of sutures and other skin closure materials. J Cosmet Laser Ther 2010;12:296302.

4. Sundaram K, Piuzzi NS, Patterson BM, et al. Skin closure with 2-octyl cyanoacrylate and polyester mesh after primary total knee arthroplasty offers superior cosmetic outcomes and patient satisfaction compared to staples: a prospective trial. Eur J Orthop Surg Traumatol 2020;30:447-53.

5. Mahalingam S, Alatsatianos A, Pitkin L, et al. Does the technique of skin closure affect the cosmesis of cervical thyroidectomy and parathyroidectomy scars? A review of literature. Facial Plast Surg 2018;34:524-8.

6. Wilson AD, Mercer N. Dermabond tissue adhesive versus Steri-Strips in unilateral cleft lip repair: an audit of infection and hypertrophic scar rates. Cleft Palate Craniofac J 2008;45:614-9.

7. Nipshagen MD, Hage JJ, Beekman WH. Use of 2-octyl-cyanoacrylate skin adhesive (Dermabond) for wound closure following reduction mammaplasty: a prospective, randomized intervention study. Plast Reconstr Surg 2008;122:10-8.

8. Fluellen S, Mackey K, Hagglund K, et al. Randomized clinical trial comparing skin closure with tissue adhesives vs subcuticular suture after robotic urogynecologic procedures. World J Methodol 2020;10:1-6.

9. Hancock NJ, Samuel AW. Use of Dermabond tissue adhesive in hand surgery. J Wound Care 2007;16:441-3.

10. Prince D, Solanki Z, Varughese R, et al. Antibacterial effect and proposed mechanism of action of a topical surgical adhesive. Am J Infect Control 2018;46:26-9. 
11. Sivrioglu N, Irkoren S, Ceylan E, et al. 2-octyl-cyanoacrylate glue for fixation of STSG in genitourinary tissue defects due to Fournier gangrene: a preliminary trial. Ulus Travma Acil Cerrahi Derg 2013;19:215-8.

12. Procter LD, Davenport DL, Bernard AC, et al. General sur- gical operative duration is associated with increased riskadjusted infectious complication rates and length of hospital stay. J Am Coll Surg 2010;210:60-5.

13. Guo S, Dipietro LA. Factors affecting wound healing. J Dent Res 2010;89:219-29. 